\title{
Requirement for hsp70 in the mitochondrial matrix for translocation and folding of precursor proteins
}

\section{Pil-Jung Kang", Joachim Ostermann", Jeffery Shilling", Walter Neupert ${ }^{\dagger}$, Elizabeth A. Craig ${ }^{*}$ \& Nikolaus Pfanner ${ }^{\dagger}$}

* Department of Physiological Chemistry, University of Wisconsin, Madison, Wisconsin 53706, USA

$\dagger$ Institut für Physiologische Chemie, Universität München, Goethestrasse 33, D-8000 München 2, FRG

By analysis of a temperature-sensitive yeast mutant, a heat-shock protein in the matrix of mitochondria, mitochondrial hsp70 (Ssc1p), is found to be involved both in translocation of nuclear-encoded precursor proteins across the mitochondrial membranes and in (re)folding of imported proteins in the matrix.

THE biogenesis of mitochondrial proteins is a multistep process $^{1-3}$ in which precursor proteins synthesized in the cytosol are recognized by specific receptors on the mitochondrial surface. The precursors are inserted into the outer membrane and are translocated through contact sites between both mitochondrial membranes in an unfolded conformation. In the matrix, amino-terminal signal sequences (presequences) of the precursors are proteolytically cleaved. The imported proteins are (re)folded and are assembled into functional complexes. An important goal in the molecular analysis of organelle biogenesis is the identification and characterization of the components that are required for mitochondrial protein import. Stress proteins or heat shock proteins (hsps) are involved in several steps of protein transfer into mitochondria.

Hsps, so named because expression of most (but not all) is increased under various cellular stress conditions, have been grouped into several families according to similarities in their primary structure ${ }^{4}$. The largest hsp family is composed of proteins of relative molecular mass about $70,000\left(M_{\mathrm{r}} \sim 70 \mathrm{~K}\right)$ (hsp70s). Cytosolic hsp70s, termed Ssa1 $4 \mathrm{p}$ in yeast ${ }^{5}$, stimulate translocation of precursor proteins into mitochondria and endoplasmic reticulum (ER), suggesting these hsp70s are involved in conferring transport-competence to precursors ${ }^{6-10}$. The $60 \mathrm{~K}$ hsp family ('chaperonins') includes mitochondrial hsp60, chloroplast Rubisco-subunit binding protein and GroEL in Escherichia coli ${ }^{11-14}$. By using transport intermediates of precursor proteins, hsp60 was shown to interact with imported proteins in an ATP-dependent reaction, thereby promoting the (re)folding and assembly of proteins, giving a first indication for a

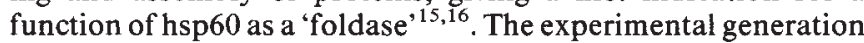
of translocation and folding intermediates of mitochondrial precursor proteins thus provides a powerful system for the analysis of two intriguing problems in cell biology, the mechanisms of intracellular protein sorting and the functions of heat shock proteins.

We recently reported that of the eight hsp70s that have been identified in the yeast Saccharomyces cerevisiae, one (Ssc1p) is located in mitochondria ${ }^{17}$. This protein performs an essential cellular function as disruption of SSC1 results in lethality ${ }^{18}$. Mitochondrial hsp70s have also been found in several other organisms ${ }^{19-22}$. To study the function of Ssc1p we isolated a temperature-sensitive $\operatorname{ssc} 1$ yeast mutant. As the mutant cells

$\ddagger$ Present address: Department of Biology, Princeton University, Princeton, New Jersey 08544-1014, USA. accumulated the precursor forms of mitochondrial proteins, we analysed the assembly pathways of mitochondrial proteins in detail and found a dual role for Ssc1p: involvement in translocation of precursor proteins through mitochondrial contact sites and in folding of proteins in the mitochondrial matrix. We believe that the homologous $\mathrm{hsp} 70 \mathrm{~s}$ in prokaryotes, eukaryotic cytosol, ER and chloroplasts may have similar functions.
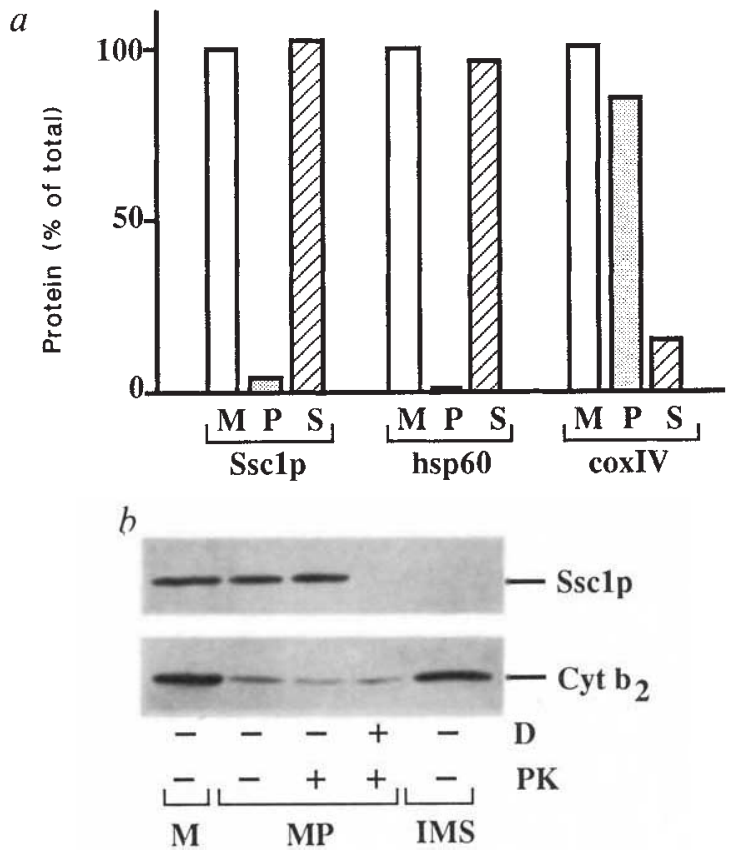

FIG. 1 Mitochondrial hsp70 (Ssc1p) is located in the matrix. a, Separation of membrane and soluble components. Mitochondrial protein $(\mathrm{M}, 30 \mu \mathrm{g})$ and the fractional equivalents of membrane pellet $(P)$ and soluble components (S) analysed by immunoblotting with antisera against: Ssc1p, hsp60 and subunit IV of cytochrome oxidase (coxIV). b, Formation of mitoplasts and release of components of the intermembrane space (IMS). Mitochondria (M, $30 \mu \mathrm{g}$ ) or the fractional equivalents of mitoplasts (MP) or soluble components (IMS) released during mitoplast formation. PK, proteinase $\mathrm{K}$; D, detergent (SDS). Antisera: Ssc1p, coxIV, cytochrome $b_{2}\left(\right.$ Cyt $\left.b_{2}\right)$.

METHODS. Mitochondria were prepared from D273-10B cells grown in semisynthetic media containing $3 \%$ glycerol and $2 \%$ lactate as described $^{17}$. $a$, Mitochondria were subjected to sonication and the membrane components separated by centrifugation for 20 min at 48,000 r.p.m. in a Beckman TLA 100.3 rotor. $b$, Mitoplasts were prepared by diluting mitochondria (10 $\mathrm{mg} \mathrm{ml}^{-1}$ in $250 \mathrm{mM}$ sucrose, $1 \mathrm{mM}$ EDTA, $10 \mathrm{mM}$ MOPS buffer, $\mathrm{pH} \mathrm{7.2)}$ fivefold in $10 \mathrm{mM}$ Tris, $\mathrm{pH} 7.4$. The mitoplasts were pelleted by centrifugation for 20 min at 37,000 r.p.m. in a Beckman TLA 100.3 rotor. The supernatant (the IMS fraction) was removed and the mitoplasts resuspended in the original volume (10 $\mathrm{mg} \mathrm{ml}^{-1}$ equivalent) of $0.1 \mathrm{M}$ mannitol, $10 \mathrm{mM}$ Tris pH 7.4. Aliquots were treated with $0.2 \mathrm{mg} \mathrm{ml}^{-1}$ of proteinase $\mathrm{K} \pm 0.2 \%$ SDS for $20 \mathrm{~min}$ at $0^{\circ} \mathrm{C}$. The indicated amounts of each fraction were separated by SDS-PAGE and subjected to immunoblot analysis. The filter-bound immunoglobulins were visualized by the use of secondary antibodies linked to alkaline phosphatase and a chromogenic substrate ${ }^{17}$. 


\section{Ssc1p is localized in the matrix}

To determine the submitochondrial localization of Ssc1p, antibodies specifically directed against $S s c 1 p$ were prepared. As the 11 carboxyl-terminal amino-acid residues of Ssc1p are not conserved in other hsp70s (ref. 17), a chemically synthesized peptide corresponding to these residues was used for immunization. The antibodies reacted with Ssc1p on two-dimensional SDS polyacrylamide gels ${ }^{17}$, but not with any other hsp70 (data not shown). Mitochondria were disrupted by sonication and the soluble and membrane fractions separated by centrifugation. Ssclp was found in the soluble fraction, as was hsp60, a soluble matrix protein (Fig. 1a). As expected, the inner membrane protein subunit IV of cytochrome oxidase was present predominantly in the membrane fraction (Fig. $1 a$ ). To distinguish between a localization of Ssclp in the intermembrane space or the matrix, the mitochondrial outer membrane was selectively broken, releasing most cytochrome $b_{2}$ from the intermembrane space (Fig. 1b). Ssclp remained associated with the mitoplasts in a location protected from proteases. Sscip only became accessible to added protease on disruption of the inner mitochondrial membrane with detergent (Fig. $1 b$ ), as was found with other mitochondrial matrix proteins ${ }^{23}$. We conclude that $\mathrm{Ssclp}$ is a soluble protein of the mitochondrial matrix.

\section{A temperature-sensitive SSC1 mutant}

To analyse the function of Ssclp we isolated temperaturesensitive (ts) yeast mutants carrying mutations in SSC1. Strain BC100 ( $G A L 1: S S C 1)$, which carries an insertion mutation in the chromosomal copy of SSC1 and a centromeric plasmid containing a wild-type SSC1 gene fused to the glucose-repressible $G A L 1$ promoter, was used in the mutant screen (Fig. 2). As $S S C 1$ is an essential gene, the strain was maintained on galactose-based media to allow $S S C 1$ expression from the $G A L 1$ promoter. Another centromeric plasmid containing SSC1 under the control of its own promoter was mutagenized in vitro with hydroxylamine and used to transform BC100 (GAL1:SSC1). Transformants were screened for growth on glucose-based media at $23^{\circ} \mathrm{C}$ and $37^{\circ} \mathrm{C}$. Those permissive for growth at $23^{\circ} \mathrm{C}$, but not $37^{\circ} \mathrm{C}$ were candidates for strains carrying ssc1 temperature-sensitive (ts) mutations. Transformants were also screened for growth at $37^{\circ} \mathrm{C}$ on galactose-based media to eliminate $S S C 1$-independent ts mutations resulting from the transformation procedure. Several tests were performed on the putative SSC1 mutant plasmids to confirm that the mutation conferring temperature sensitivity was in the $S S C 1$ gene (see Fig. 2). One plasmid carrying a mutation in the protein-coding region was chosen for further analysis. Although ssc1 cells carrying the temperature-sensitive mutant ssc1 (BC100(ssc1-2)) grew well at $23^{\circ} \mathrm{C}$, after transfer to $37^{\circ} \mathrm{C}$ the growth rate rapidly decreased, and growth ceased before one doubling had occurred. The $s s c 1-2$ mutation is recessive; ssc1-2/SSC1 heterozygotes have wild-type growth rates at $23^{\circ} \mathrm{C}$ and $37^{\circ} \mathrm{C}$ (data not shown).

To test whether a defect in $S S C 1$ results in a defect in import of proteins into mitochondria, protein extracts were prepared from $s s c 1$ cells carrying the wild-type (BC100(SSC1)) or mutant gene $(\mathrm{BC} 100(s s c 1-2)$ ) (subsequently referred to as 'wild-type' and 'mutant', respectively) and analysed by immunodecoration with antibodies against nuclear-encoded mitochondrial proteins (Fig. $3 a$ ). Mutant cells shifted to non-permissive temperature accumulated the precursor forms of hsp60 (Fig. $3 a$ ), Ssclp itself and $F_{1}-A T P a s e$ subunit $\beta\left(F_{1} \beta\right)$ (data not shown). Little or no precursor was detected at permissive temperature. As a control, temperature-sensitive mif1 cells, that are known to be defective in translocation and processing of precursor proteins (M. Y. Cheng and A. L. Horwich, personal communication $)^{2,24}$, showed a similar accumulation of precursor proteins on shift to nonpermissive temperature. As expected, little or no precursor accumulated in cells carrying the wild-type $S S C 1$ gene at $23^{\circ} \mathrm{C}$ or after shift to $37^{\circ} \mathrm{C}$ (Fig. $3 a$ ).

By pulse-labelling of cells with $\left[{ }^{35} \mathrm{~S}\right]$ methionine, we could determine whether the defect in the import of precursors occurred soon after the shift to non-permissive temperature. BC100(ssc1-2) cells and mif1 cells, radiolabelled 10 min after shift, showed a massive accumulation of precursor proteins of $F_{1} \beta$, Ssclp (Fig. $3 b$ ) and hsp60 (data not shown). Unlike the mif1 cells, BC100(ssc1-2) cells labelled at $23^{\circ} \mathrm{C}$ accumulated a small amount of precursor, indicating a slight defect of protein

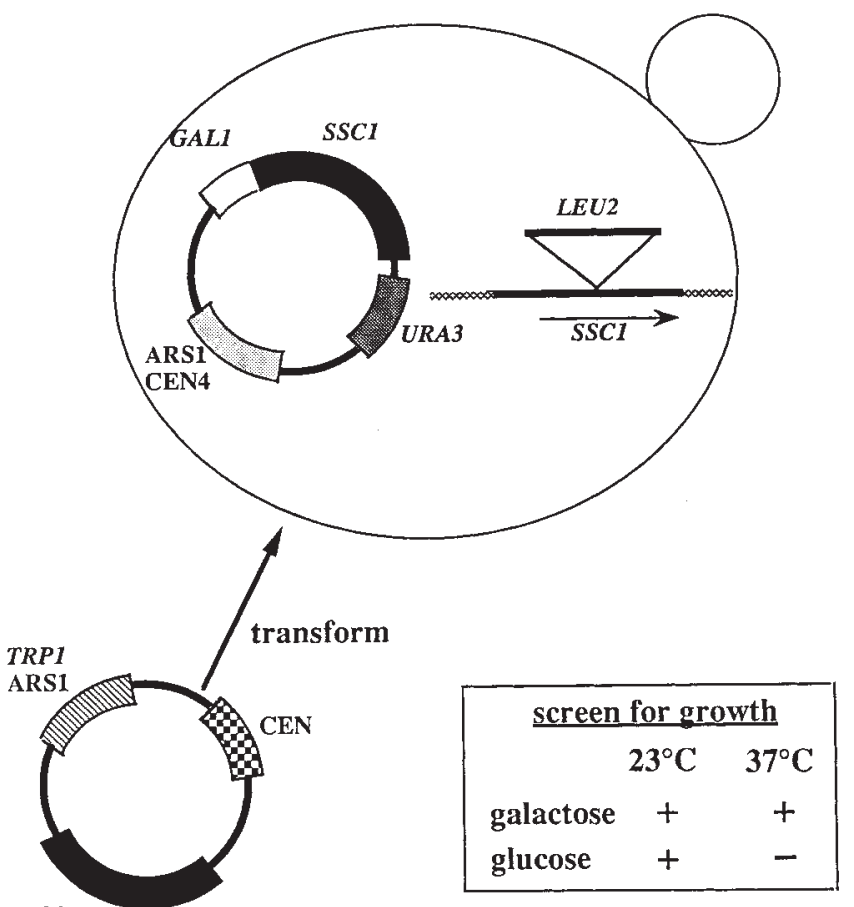

FIG. 2 Screen for temperature-sensitive $s s c 1$ yeast mutants. Strain BC100(GAL1:SSC1)(MAT a leu2-3,112 Atrp1 lys2 ura3-52 ssc1-1 pGAL1:SSC1(URA3)) was used. The ssc1-1 allele is a disruption mutation constructed by inserting the LEU2 gene into the Sall site in the codon of amino acid 318 in the protein-coding region of SSC1. The GAL1: SSC1 fusion was constructed by ligating plasmid pMB272 (a gift from Mark Johnston) with a 2.3 kilobase (kb) BamHI-Bg/ll fragment containing the SSC1 gene with a BamH1 linker at position -70 (with +1 being the $A$ of the initiating ATG) ${ }^{17}$. A plasmid (pJK808A) carrying a $3.2 \mathrm{~kb}$ Xbal-Bg/ll fragment that contained the entire SSC1 gene with its own promoter in the centromeric vector, pYEcen30 (CEN3, ARS1 and TRP1), was treated with $1 \mathrm{M}$ hydroxylamine at $37^{\circ} \mathrm{C}$ for $24 \mathrm{~h}$. The plasmid mutagenized in vitro was used to transform BC10O(GAL1: SSC1). Transformants were screened for temperature-sensitive growth at $37^{\circ} \mathrm{C}$ on YPD (1\% Bacto-yeast extract, $1 \%$ Bacto-peptone, $2 \%$ dextrose) plates. Plasmids were recovered from the temperature-sensitive transformants and used to transform strain $\mathrm{BC} 100$ (GAL1:SSC1) to confirm that the temperature-sensitive growth on glucose-based media was caused by the plasmid. Segments of the candidates plasmids were recloned into unmutagenized pJK808A to determine if the mutation(s) responsible for the ts growth phenotype were in the SSC1 protein coding region. One of the SSC1 genes with a mutation(s) within a 1.4-kb Clal fragment (between the codons for amino acids 35-501) was chosen for further analysis and named SSC1-2. The pGAL1(SSC1(URA3)) plasmid was cured from strain $\mathrm{BC100}(G A L 1$ :SSC1; ssC1-2) to give BC100(sSC1-2). Strain BC10O(GAL1:SSC1) was transformed with unmutagenized $\mathrm{pJK} 808$ and then transformants were cured of pGAL1(SSC1(URA3)) to give the control strain BC100(SSC1).

Hydroxylamine-treated

SSC1 plasmid 
import even under permissive growth conditions. As expected, no labelled precursor was found in $\mathrm{BC} 100(S S C 1)$ cells at $23{ }^{\circ} \mathrm{C}$ or $37^{\circ} \mathrm{C}$. Together these results indicate a defect in import of several mitochondrial precursor proteins in $\mathrm{ssc1}^{-}$ cells.

To determine the localization of the accumulated precursor in the mutant cells, fractionation experiments were carried out. Almost all the precursor of $F_{1} \beta$ labelled after shift to nonpermissive temperature was present in the mitochondrial fraction (Fig. $3 c$ ). Unlike the mature form in wild-type cells, the precursor associated with the mutant mitochondria was accessible to added protease (Fig. $3 d$ ). These results suggest that inactivation of Ssclp leads to a block in mitochondrial protein import.

\section{Ssc1p in protein translocation}

The accumulation of precursor proteins in vivo suggests that one or more step(s) in the import and assembly pathway of mitochondrial proteins depend(s) on functional Ssc1p. However, as in the analysis of other yeast mutants in vivo ${ }^{15,24-26}$, this finding per se does not allow determination of which step actually requires $S s c 1 p$ and if the putative role of $S s c 1 p$ in protein import is a direct or indirect one.

A detailed characterization of the role of Ssclp in import of precursor proteins required in vitro analysis. For these studies, precursor proteins were synthesized in rabbit reticulocyte lysates in the presence of $\left[{ }^{35} \mathrm{~S}\right]$ methionine. Mitochondria were isolated from ssc1 cells carrying the wild-type or mutant SSC1 gene and preincubated for $10 \mathrm{~min}$ at $37^{\circ} \mathrm{C}$. Import of the precursors into mitochondria was performed at $25^{\circ} \mathrm{C}$ and assessed by the proteolytic processing of the precursors and the protection of the imported proteins against externally added proteinase $\mathrm{K}^{23,27,28}$.

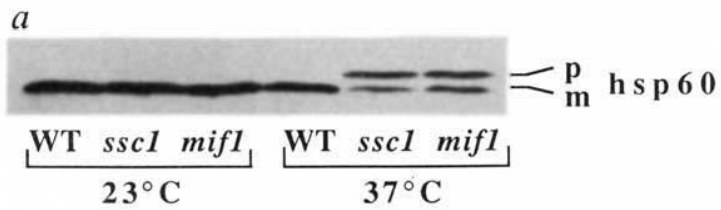

$b$

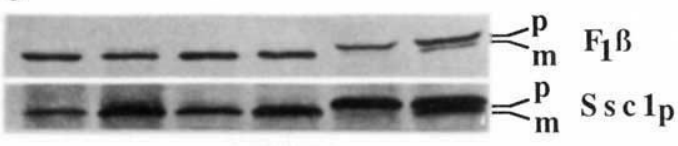

WT ssc1 mif1, WT ssc1 mif1,

$23^{\circ} \mathrm{C}$

$37^{\circ} \mathrm{C}$

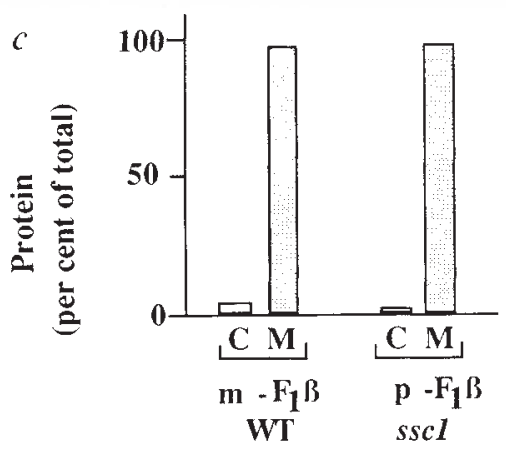

$d$

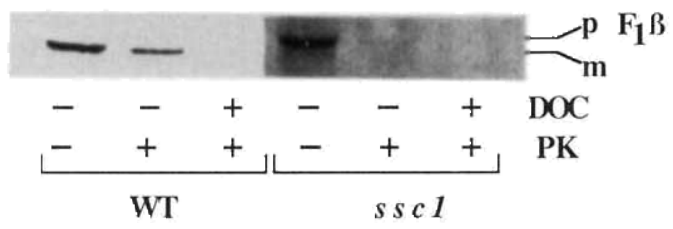

Import of the precursor of cytochrome $b_{2}$ into the mutant mitochondria (Fig. $4 a$ ). The total amount of precursor in the mitochondria was strongly decreased compared with wild-type import reactions (in the absence of proteinase $\mathrm{K}$ ) was not reduced after incubation with the mutant mitochondria, excluding unspecific degradation of precursor (see below).

The translocation of precursor proteins across the two mitochondrial membranes can be divided experimentally into two subsequent reactions ${ }^{28-33}$. First, the precursor protein is inserted into mitochondrial contact sites such that the aminoterminal presequence reaches the matrix space where it is proteolytically cleaved, while a major portion of the precursor is still located on the cytosolic side. Second, the remainder of the precursor is unfolded and also translocated through contact sites, leading to complete translocation of the precursor into the matrix. Only the initial insertion step, but not the completion of translocation, depends on the membrane potential $\Delta \Psi$ across the inner membrane. We used the precursors of $F_{1} \beta$ and of the $\mathrm{Fe} / \mathrm{S}$ protein of the $\mathrm{bc}_{1}$-complex ${ }^{29,30,33}$ to analyse which of the two transport steps was preferentially affected by the mutational alteration of $\mathrm{Ssc1p}$. The precursor proteins were efficiently inserted into the mutant mitochondria and specifically processed by the matrix processing peptidase (Fig. $4 b$ ). Complete translocation of the precursors to a protease-protected location, however, was impaired in the mutant mitochondria, indicating accumulation of the precursor proteins in contact sites, thereby spanning the outer and inner membranes. Similarly, a hybrid protein beween the presequence of $\mathrm{F}_{0}$-ATPase subunit 9 and complete dihydrofolate reductase (Su9-DHFR) ${ }^{16,31,34}$ was accumulated in contact sites of the mutant mitochondria (Fig. $4 c$ ). Thus, the $\Delta \Psi$-independent completion of translocation is defective in the mutant mitochondria. These results exclude the trivial possibility

FIG. 3 Accumulation of mitochondrial precursor proteins in yeast cells with defective Ssc1p. a, Immunoblot analysis of mitochondrial protein hsp60 in ssc1 mutant cells. WT, wild-type (BC100(SSC1)); ssc1, mutant (BC100( ssc12)). $23^{\circ} \mathrm{C}$, cells grown and maintained at $23^{\circ} \mathrm{C} ; 37^{\circ} \mathrm{C}$, cells grown at $23^{\circ} \mathrm{C}$ and shifted to $37{ }^{\circ} \mathrm{C}$ before harvest. $\mathrm{p}$. Precursor; $\mathrm{m}$, mature-sized protein. $b$. Pulse labelling of proteins in mutant cells. Immunoprecipitation of radiolabelled $F_{1} \beta$ and Ssc1p. $c$, Separation of mitochondrial and cytoplasmic fractions. $C$, cytoplasmic fraction; $M$, mitochondrial fraction. $d$, Proteaseaccessibility of accumulated precursor. PK, proteinase K; DOC, deoxycholate. METHODS. a, WT (BC100(SSC1)), ssc1 (mutant; BC100(ssc1-2)) and mif1 cells were grown in YPD media at $23^{\circ} \mathrm{C}$. At the early log phase $\left(O D_{600} \sim 1.0\right)$, the cultures were divided; one aliquot remained at $23^{\circ} \mathrm{C}$, the other was shifted from $23^{\circ} \mathrm{C}$ to $37^{\circ} \mathrm{C}$. One hour after temperature-shift, $20 \mathrm{OD}_{600}$ units of cells were harvested and protein extracts were prepared by glass bead lysis ${ }^{58}$. Protein from one $\mathrm{OD}_{600}$ unit of cells were loaded on a SDS $(7.5 \%)$ polyacrylamide gel, electrophoresed, transferred to immobilon membranes (Millipore) and immunoblotted with antisera directed against hsp60. $b$, Cells were grown at $23^{\circ} \mathrm{C}$ in selective minimal media $(0.67 \%$ Bacto-yeast nitrogen base, $2 \%$ glucose) lacking methionine. During early log phase (at $\mathrm{OD}_{600} \sim 1.0$ ), $10 \mathrm{OD}$-units of cells were collected and resuspended in $1 \mathrm{ml}$ of fresh labelling medium. After 10 -min preincubation at $23^{\circ} \mathrm{C}$ or $37^{\circ} \mathrm{C}$, $\left.{ }^{35} \mathrm{~S}\right]$ methionine $\left(100 \mu \mathrm{Ci} \mathrm{ml}{ }^{-1}\right)$ was added. After $10 \mathrm{~min}$, cells were put on ice and $\mathrm{NaN}_{3}$ and cycloheximide added to $10 \mathrm{mM}$ and $100 \mu \mathrm{g} \mathrm{ml} l^{-1}$, respectively. Protein extracts were prepared. Aliquots containing $\sim 1 \times 10^{6}$ c.p.m. were adjusted to $0.5 \%$ SDS and immunoprecipitated ${ }^{59}$ using antibodies specific for $F_{1} \beta$ or Ssc1p and protein A-Sepharose, $c$, Cells were grown as described in $b$. Five $\mathrm{OD}_{600}$ units of cells were harvested, resuspended in $2 \mathrm{ml}$ of fresh glucose-based media and shifted to $37^{\circ} \mathrm{C}$. After $30 \mathrm{~min}$ $400 \mu \mathrm{Ci}\left[{ }^{35} \mathrm{~S}\right]$ methionine was added and incubation carried out for an additional $30 \mathrm{~min}$. The labelling was terminated by addition of $\mathrm{NaN}_{3}$ and $20 \mathrm{OD}_{600}$ units of unlabelled cells were added as carrier. After preparation of spheroplasts, mitochondrial and cytoplasmic fractions were prepared ${ }^{15,23}$. Equivalent amounts of cytoplasmic and mitochondrial fractions were immunoprecipitated using $F_{1} \beta$-specific antibodies as described in $b$. $d$, Mitochondrial fractions prepared from cells labelled from 30-60 min after shift to $37^{\circ} \mathrm{C}$ as described in $c$, were treated with proteinase $\mathrm{K}\left(230 \mu \mathrm{g} \mathrm{ml}^{-1}\right)$ in the presence or absence of $0.45 \%$ deoxycholate (DOC) for $5 \mathrm{~min}$ at $0^{\circ} \mathrm{C}$. Aliquots $\left(0.5 \times 10^{6}\right.$ c.p.m., WT; $2 \times 10^{6}$ c.p.m., ssc1-2) were immunoprecipitated using $F_{1} \beta$-specific antibody. 
FIG. 4 Deficiency of Ssc1p impairs protein translocation through mitochondrial contact sites. a, Kinetics of import of in vitro-synthesized cytochrome $b_{2}$ into isolated mitochondria. $b$, Accumulation of the precursors of $F_{1} \beta$ amounts of total processed precursor proteins and of protease-protected processed proteins are compared. $c$, Accumulation of Su9-DHFR in contact sites. Kinetics of processing and transport to a protease-protected location of the fusion protein Su9-DHFR. $d$, Ureadenatured Su9-DHFR is efficiently imported. WT, wildtype mitochondria (BC10O(SSC1)); ssc1, mutant mitochondria (BC100(ssc1-2)); PK, proteinase $K ; p, i, m$, precursor-, intermediate- and mature-sized forms of a protein, respectively.

METHODS. Wild-type (BC10O(SSC1)) and ssc1-2 cells were grown on YPG medium for $20 \mathrm{~h}$ at $23^{\circ} \mathrm{C}$. Exponentially growing cells were collected and mitochondria were isolated as described ${ }^{23}$. Precursor proteins were synthesized in rabbit reticulocyte lysates by coupled transcription/translation and labelled with $\left[{ }^{35} \mathrm{~S}\right.$ ]methionine ${ }^{27}$. Mitochondria $\left(0.25 \mathrm{mg}\right.$ protein $\mathrm{ml}^{-1}$ ) were preincubated for $10 \mathrm{~min}$ at $37^{\circ} \mathrm{C}$ and $3 \mathrm{~min}$ at $25^{\circ} \mathrm{C}$ in import buffer ( $3 \% \mathrm{BSA}, 220 \mathrm{mM}$ sucrose, $70 \mathrm{mM}$ $\mathrm{KCl}, 2.5 \mathrm{mM} \mathrm{MgCl}_{2}, 10 \mathrm{mM}$ MOPS, pH 7.2). Reticulocyte lysate $(10 \%(\mathrm{v} / \mathrm{v}))$ containing the indicated radiolabelled precursor proteins, NADH ( $2 \mathrm{mM})$ and ATP $(1 \mathrm{mM})$ were added and the import reaction was incubated at $25^{\circ} \mathrm{C}$. a, Aliquots (containing precursor of cytochrome $b_{2}$ ) were withdrawn at the indicated time, diluted threefold in ice-cold SEM buffer (250 mM sucrose, $1 \mathrm{mM}$ EDTA $10 \mathrm{mM}$ MOPS, $\mathrm{pH}$ 7.2) and incubated with proteinase $\mathrm{K}$ $\left(20 \mu \mathrm{g} \mathrm{ml}^{-1}\right)$ for $15 \mathrm{~min}$ at $0^{\circ} \mathrm{C}$. After addition of $1 \mathrm{mM}$ PMSF, mitochondria were reisolated by centrifugation and analysed by SDS-PAGE and fluorography. $b$, Aliquots (containing precursors of $\mathrm{F}_{1} \beta$ and $\mathrm{Fe} / \mathrm{S}$ protein) were withdrawn after $20 \mathrm{~min}$ and incubated at $0{ }^{\circ} \mathrm{C}$ in the presence or absence of proteinase $\mathrm{K}$. Mitochondria were reisolated and analysed as described above. The fluorographs (inserts) were quantified by laser densitometry (columns). The amount of processed protein in wild-type mitochondria was taken as $100 \%$. c, Aliquots (containing precursor of SU9-DHFR) were withdrawn at the indicated times and further treated as described above. $d$, Reticulocyte lysate containing SU9-DHFR was precipitated with ammonium sulphate at $66 \%$ saturation and dissolved in $8 \mathrm{M}$ urea, $20 \mathrm{mM}$ Tris, $\mathrm{pH} 7.5$ (ref. 16). The urea-denatured precursor was diluted 15 -fold into the import reaction. After incubation for the indicated times at $25^{\circ} \mathrm{C}$, aliquots were treated with proteinase $\mathrm{K}$. The mitochondria were reisolated and analysed as described above. The amount of imported protein is expressed as the percentage of total added precursor protein. The mutant mitochondria contained $98-104 \%$ of the wildtype levels of marker proteins, cytochrome $c$, cytochrome $b_{2}$ and fumarase, under the various import conditions (presence and absence of proteinase $\mathrm{K}$ ), confirming the intactness of mitochondrial outer and inner membranes as described ${ }^{23,33}$. and $\mathrm{Fe} / \mathrm{S}$ protein in mitochondrial contact sites. The
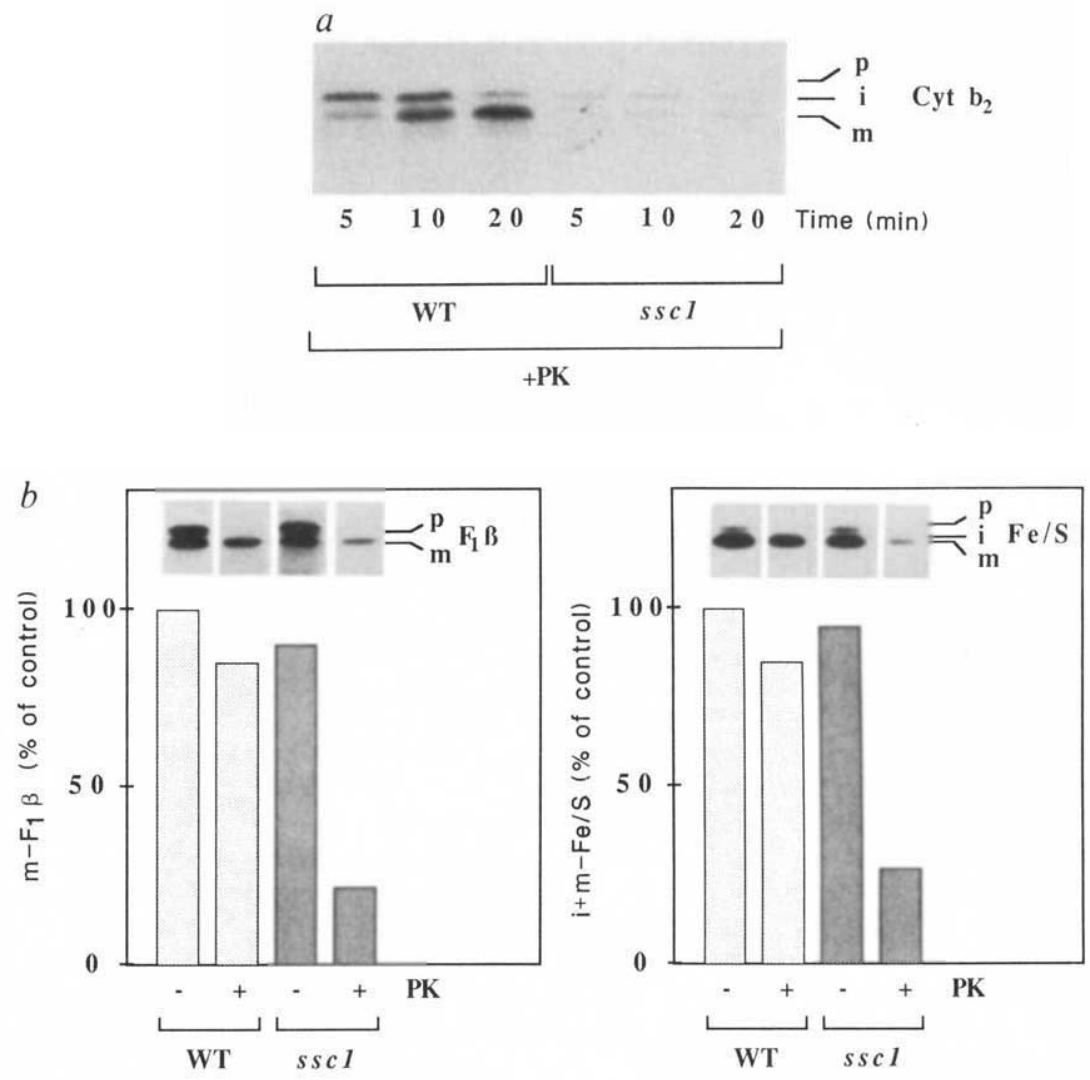

c

$-\mathrm{PK}$

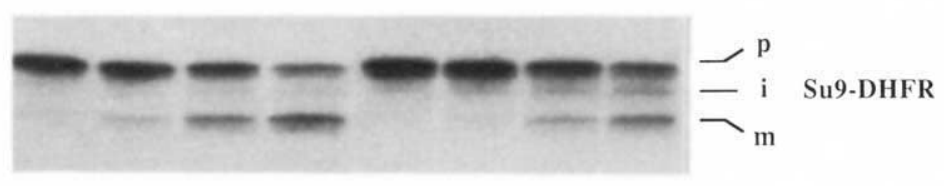

+ PK
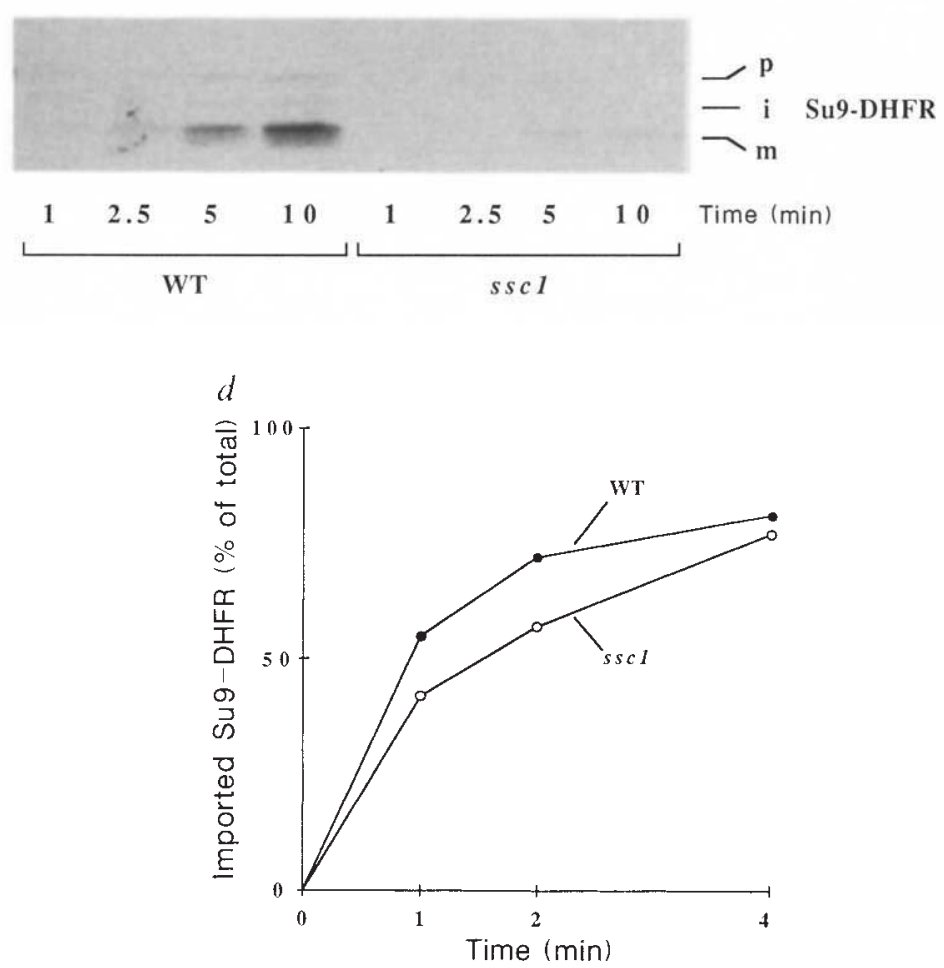


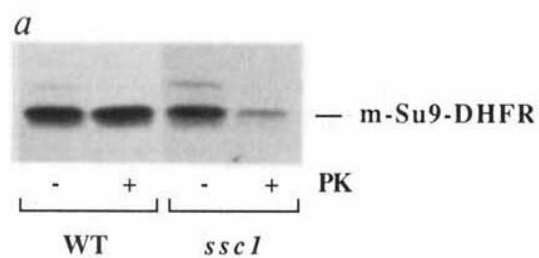

FIG. 5 Accumulation of unfolded precursor protein in the mutant mitochondria. Urea-denatured Su9-DHFR was imported into isolated wild-type (WT) and ssc1-2 mitochondria. The amount of protease-resistant (folded) protein on lysis of the mitochondrial membranes with detergent is compared. PK proteinase $\mathrm{K}$.

METHODS. Import of urea-denatured SU9-DHFR for $4 \mathrm{~min}(a)$ or the indicated times $(b)$ and incubation of the mitochondria with proteinase $K$ was performed as described in the legend to Fig. 4 (ref. 16). Mitochondria were reisolated, resuspended in SEM buffer containing $0.4 \%$ digitonin and divided into halves. One half $(+\mathrm{PK})$ received proteinase $\mathrm{K}\left(10 \mu \mathrm{g} \mathrm{m} \mathrm{l}^{-1}\right)$ and was incubated for $10 \mathrm{~min}$ on ice. Trichloroacetic acid $(10 \%)$ was added to each aliquot and the precipitated proteins were analysed by SDS-PAGE, fluorography and laser-densitometry. $b$, The amount of protease-resistant protein

that the translocation defect might be due to a diminished membrane potential.

As the completion of membrane translocation includes the unfolding of large parts of the precursors on the cytosolic side, we reasoned that an artificial unfolding of precursors should reduce the translocation defect into the mutant mitochondria. The hybrid protein Su9-DHFR was thought to be a suitable substrate for an analysis of this problem as we had previously found that Su9-DHFR could be transported into mitochondria after denaturation by a preincubation in $8 \mathrm{M}$ urea and subsequent dilution into the import reaction ${ }^{16}$. Su9-DHFR denatured with urea was efficiently imported into the mutant mitochondria at rates that were close to the import rates observed with wild-type mitochondria (Fig. $4 d$ ), in contrast to the results obtained with the partially folded Su9-DHFR synthesized in reticulocyte lysate (Fig. $4 c$ ). This difference suggests an involvement of Ssc1p in unfolding of the precursor and confirms that the transport defect is not related to the $\Delta \Psi$-dependent step. Moreover, it excludes the possibility that the decreased amounts of protease-protected proteins observed in mutant mitochondria (Fig. $4 a-c$ ) are caused by an increased susceptibility of mutant mitochondria to proteolytic attack. The intactness of the mitochondria was also confirmed by the analysis of several marker proteins (see Fig. 4 legend). We conclude that Ssclp is required for the completion of transport of precursor proteins through mitochondrial contact sites, including the unfolding of a portion of the precursor on the cytosolic side. The accumulation of unprocessed precursors in vivo (Fig. 3) is probably due to saturation of import sites by the massive amounts of arrested precursors, thereby leading to a 'back-up' block at an earlier import stage.

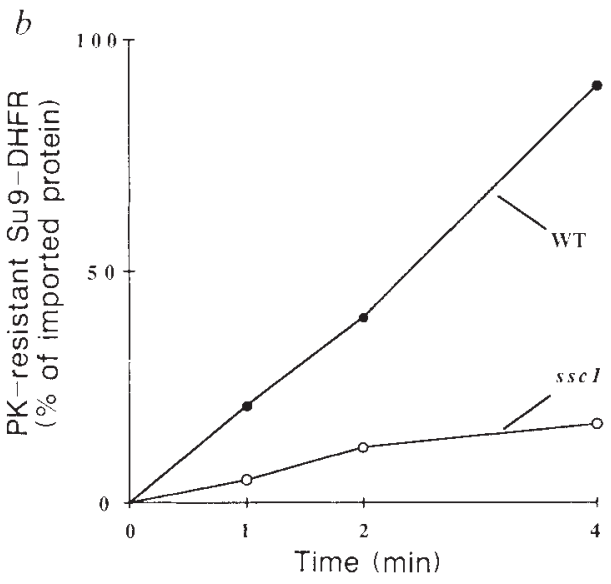

(treatment with proteinase $\mathrm{K}$ on lysis of mitochondria with digitonin) is given as percentage of total imported protein (protected against proteinase $\mathrm{K}$ in intact mitochondria) at each time point.

\section{Ssc1p in (re)folding of imported proteins}

As artificial denaturation of precursors allowed a circumvention of the translocation defect, it was possible to investigate if further import steps occurring in the matrix would be affected in the mutant mitochondria. Of particular importance is the refolding of imported polypeptide chains in the matrix, which was previously shown to involve mitochondrial hsp60 (ref. 16). For the experimental analysis, the hybrid protein Su9-DHFR was used as the folding state of its DHFR domain can be investigated by assaying its sensitivity towards proteolytic attack. After import of Su9-DHFR into mitochondria and removal of its presequence by the processing peptidase, the mitochondria were lysed with detergent and treated with proteinase $\mathrm{K}$ : the unfolded protein was sensitive to proteolytic digestion whereas the folded polypeptide (essentially consisting of mature DHFR) exhibited a high protease resistance ${ }^{16}$. In the experiment described in Fig. 5 , we found that Su9-DHFR was highly protease-sensitive after import into the mutant mitochondria, indicating that it did not achieve the tightly folded conformation. In wild-type mitochondria, the protein became folded and thus protease-resistant (Fig. 5 ). We conclude that the (re)folding of proteins in the mitochondrial matrix requires functional $S$ sc1p.

Ssclp could be directly involved in translocation and refolding of precursor proteins by a physical interaction with the precursors. Alternatively, the defects observed in the mutant mitochondria could be indirect, because of an impairment of components of the import machinery by mutated Ssclp. A direct interaction of precursor proteins with Ssclp would demonstrate the first, more interesting possibility. As such a complex would be a transient intermediate, the reaction could be demonstrated only by selectively blocking release of precursors from $\mathrm{Ssc1p}$. To see
FIG. 6 Association of precursor proteins with Ssc1p. Fe/S protein and urea-denatured Su9DHFR imported into mutant mitochondria (ssc1) are co-precipitated with antibodies specific for Ssc1p on lysis of mitochondria with detergent. WT, wild-type mitochondria.

METHODS. Fe/S protein and urea-denatured Su9DHFR were imported into isolated wild-type (WT) and ssc1-2 (ssc1) mitochondria as described in the legend of Fig. 4. Immunoglobulin G from $15 \mu \mathrm{l}$ rabbit antisera specific for $\mathrm{Fe} / \mathrm{S}$ protein, DHFR or Ssc1p. or pre-immune serum were pre-bound to protein A-Sepharose. Mitochondria $(15 \mu \mathrm{g}$ of protein per sample) were dissolved in $0.1 \%$ Triton $X-100,100 \mathrm{mM} \mathrm{NaCl}$, $10 \mathrm{mM}$ Tris, $\mathrm{pH} 7.5(150 \mu \mathrm{l})$ and incubated with the protein A-Sepharose under gentle mixing for $1 \mathrm{~h}$ at $4^{\circ} \mathrm{C}$. The immunoprecipitates were
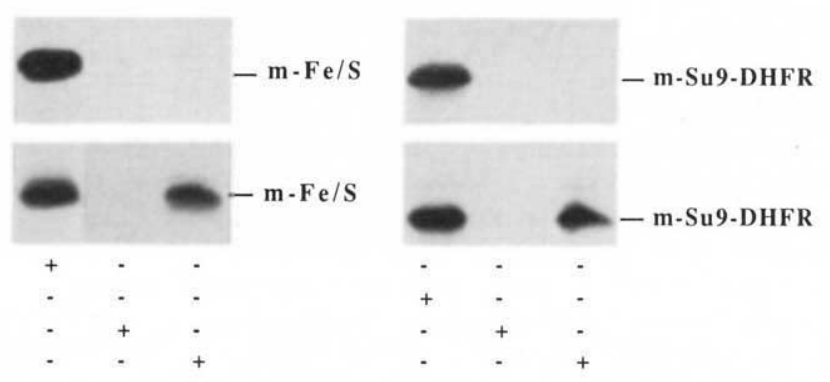

washed twice in the buffer described above and once in $10 \mathrm{mM}$ Tris, $\mathrm{pH} 7.5$, dissolved in SDS-containing buffer and analysed by SDS-PAGE and fluorography. 
if the mutation of Ssclp led to impairment of a step(s) required for release of precursors without abolishing the initial interaction of precursors with Ssclp, we tried to purify a complex between precursor proteins and mutant Ssc1p. Using the antibodies specifically directed against the carboxyl-terminus of Ssclp, we coprecipitated imported $\mathrm{Fe} / \mathrm{S}$ protein and $\mathrm{Su} 9-\mathrm{DHFR}$ after lysis of mitochondria with detergent (Fig. 6). This co-immunoprecipitation provides strong support for a physical interaction of the precursors with Ssc1p and suggests a direct involvement of Ssc1p in mitochondrial protein import. In our control experiment, $\mathrm{Fe} / \mathrm{S}$ protein and Su9-DHFR in wild-type mitochondria were fully translocated and folded ${ }^{16,30,34}$, and thus not co-precipitated with antibodies against Ssc1p (Fig. 6).

\section{Discussion}

Hsp70 (Ssc1p) in the mitochondrial matrix is required for import of precursor proteins into mitochondria. Specifically, completion of protein transport through contact sites, including unfolding and membrane translocation of the main parts of the precursor proteins, and refolding of imported proteins in the matrix, depend on functional Ssclp. The isolation of a complex between precursor proteins and Ssclp suggests a direct interaction of Ssclp with precursor proteins.

We propose that $\mathrm{Ssclp}$ mediates between the translocation apparatus in contact sites and the folding machinery in the matrix of mitochondria. Ssc1p would bind the precursor protein as it emerges on the matrix side of contact sites and thereby support the completion of translocation, including facilitation of unfolding of the polypeptide chain by the import apparatus on the cytosolic side. By interaction with one or more Ssc1p molecule(s) the precursor would be 'pulled' towards the inside of mitochondria, perhaps thereby facilitating the unfolding reaction. In addition, tight binding of the precursor in transit to Ssc1p's could render the translocation vectorial and thus assist the completion of membrane transport. The energy requirement for complete unfolding of many proteins was found to be as low as $5-10 \mathrm{kcal} \mathrm{mol}^{-1}$ (ref. 35). Therefore, it is conceivable that the free energy that might be released by binding of Ssc1p molecules to a polypeptide chain appearing on the matrix side may be considerably larger than the energy required for unfolding of the same polypeptide chain. The exact molecular mechanism of the role of Ssclp will remain elusive until other components of the unfolding and translocation apparatus of contact sites have been identified. Second, Ssc1p seems to be an initial component in the chain of reactions leading to refolding of imported proteins. We previously reported that the refolding reaction per se could occur in association with hsp60 in the matrix ${ }^{16}$. Ssclp could directly participate in this refolding process. Now, as Ssc1p seems to be a binding protein for precursors in transit through contact sites, we prefer a model in which Ssclp accepts the incoming precursors, keeps them in an unfolded conformation and possibly mediates their transfer to hsp60. The properties of Ssc1p, as revealed by analysis of the yeast mutant, group it into the class of proteins collectively termed 'molecular chaperones"14,36,37 or "polypeptide chain binding proteins'38, that mediate the folding and assembly of other polypeptides.

The completion of protein transfer through mitochondrial contact sites does not depend on the addition of an external energy source (when one round of translocation is analysed) ${ }^{39}$. The binding of precursor proteins to mitochondrial hsp70 and possibly also hsp60 could thus provide the energy for membrane translocation. Hydrolysis of ATP might then be required for releasing the precursor proteins from the chaperones, setting the chaperones free for new rounds of transport.

As the previously described hsp70s (cytosolic hsp70s, BiP in the ER, the DnaK protein in E. coli and probably also chloroplast hsp $70 \mathrm{~s}^{22,40}$ ) are very similar to Sscl $\mathrm{p}^{17,19,41-43}$, it is tempting to speculate that they perform functions similar to those of mitochondrial hsp70. The proposed role of cytosolic hsp70s in maintaining precursor proteins in an unfolded conformation, supporting the translocation of proteins into organelle membranes ${ }^{6-10,44,45}$, agrees with the indicated functions of Ssclp. BiP has been strongly implicated in the assembly of protein complexes in the lumen of the $\mathrm{ER}^{9,37,43,46-51}$. BiP could be involved in protein translocation ${ }^{52}$; but such a role has been questioned $^{51,53}$. The results reported here would support the model that $\mathrm{BiP}$ may be required at early stages, for translocation of proteins into the ER and for the reactions leading to refolding of imported proteins. Such a function of hsp $70 \mathrm{~s}$ is consistent with the finding ${ }^{54}$ that both cytosolic hsp70s and BiP can interact with peptides that might resemble structures of loosely folded precursor proteins and agrees with the proposal ${ }^{38,45}$ that polypeptide chains emerging on the surface of ribosomes or the non-cytosolic surface of organelle membranes will interact productively with hsp70s. Phillips and Silhavy ${ }^{55}$ found that DnaK, when overexpressed, stimulates protein export in E. coli. Biochemical studies on a role of DnaK in modulating the conformation of precursor proteins seem to be promising, particularly as Ssclp is more similar to DnaK than it is to any eukaryotic hsp 70 (ref. 17). As the mechanisms of mitochondrial and chloroplast protein uptake are rather similar ${ }^{56,57}$, chloroplast hsp $70 \mathrm{~s}^{22,40}$, which have not yet been functionally characterized, could perform a function equivalent to that of mitochondrial hsp 70 . Analysis of the function of Ssclp by using translocation intermediates of mitochondrial precursor proteins thus has implications for a number of systems involved in the transport, folding and assembly of proteins.

Received 2 July; accepted 31 August 1990.

. Attardi, G. \& Schatz, G. A. Rev. Cell Biol, 4, 289-333 (1988)

2. Hartl, F.U. \& Neupert W. Science 247, 930-938 (1990)

3. Pfanner, N. \& Neupert. W. A. Rev Biochem 59, 331-353 (1990)

4. Lindquist. S. \& Craig. E. A. A. Rev. Genet 22, 631-677 (1988)

5. Werner-Washburne, M., Stone, D. E. \& Craig, E. A. Molec. cell. Biol. 7, 2568-2577 (1987).

6. Deshaies, R. J., Koch, B. D., Werner-Washburne, M., Craig, E. A. \& Schekman, R. Nature 332 800-805 (1988)

7. Murakami, H., Pain, D. \& Biobet, G. J. Cell Biol. 107, 2051-2057 (1988).

8. Chirico, W. J. Waters M. G. \& Blobel G. Nature 332, 805-810 (1988).

9. Pelham, $H$. Nature 332, 776-777 (1988).

10. Zimmermann, R., Sagstetter, M., Lewis, M. J. \& Pelham, H. R. B. EMBO J. 7, 2875-2880 (1988)

11. Hendrix, R. W. I. molec. Biol. 129, 375-392 (1979).

12. Hemmingsen, S. M. et al. Nature $\mathbf{3 3 3}, 330-334$ (1988)

13. Reading, D. S., Hallberg, R. L. \& Myers, A. M. Nature 337, 655-659 (1989)

14. Ellis, R. J. \& Hemmingsen, S. M. Trends biochem. Sci. 14, 339-342 (1989)

15. Cheng, M. Y. et al. Nature 337, 620-625 (1989).

16. Ostermann, J. Horwich, A. L. Neupert. W. \& Hartl, F.-U. Nature 341, 125-130 (1989).

17. Craig, E. A. et al. Molec. cell Biol, 9, 3000-3008 (1989).

18. Craig, E. A., Kramer, J. \& Kosic-Smithers, J. Proc. natn. Acad. Sci. U.S. A. 84, 4156-4160 (1987)

19. Engman, D. M., Kirchhoff, L. V. \& Donelson, J. E. Molec cell. Biol. 9, 5163-5168 (1989)

20. Leustek, T., Dalie, B., Amir-Shapira, D., Brot, N. \& Weissbach, H. Proc. natn. Acad. Sci. U.S.A. 86 7805-7808 (1989)

21. Mizzen, L. A., Chang, C., Garrels. J. I. \& Welch, W. J. J. biol. Chem. 264, 20664-20675 (1989).

22. Amir-Shapira, D., Leustek, T., Dalie, B., Weissbach, H. \& Brot, N. Proc. natn. Acad. Sci. U.S.A. 87, $1749-1752(1990)$

23. Hartl, F.U. Ostermann, J Guiard B. \& Neupert, W. Cell 51, 1027-1037 (1987)

24. Pollock. R. A. et al, EMBO J. 7, 3493-3500 (1988)

25. Yaffe, M. P., Ohta, S. \& Schatz, G. EMBO \&. 4, 2069-2074 (1985)

26. Hartl, F.-U., Pfanner, N., Nicholson, D. \& Neupert, W. Biochim. biophys. Acta $988,1-45$ (1989).

27. Pfaller, R., Steger, H. F., Rassow, J., Pfanner, N. \& Neupert, W. J. Cell. Biol. 107, 2483-2490 (1988)

28. Rassow, J. et al., J. Cell Biol. 109, 1421-1428 (1989).

29. Schleyer M \& Neupert W Cell 43,339-350 (1985).

30. Hartl. F.-U., Schmidt. B., Wachter, E., Weiss. H. \& Neupert, W. Cell 47, 939-951 (1986).

31. Pfanner, N., Tropschug, M. \& Neupert, W. Cell 49, 815-823 (1987).

32. Pfanner, N., Hartl, F.-U., Guiard, B. \& Neupert, W. Eur. J. Biochem. 169, 289-293 (1987)

33. Schwaiger, M., Herzog, V. \& Neupert, W. I. Cell Biol. 105, 235-246 (1987).

34. Pfanner, N., Müller, H. K., Harmey, M. A. \& Neupert, W. EMBO J. 6, 3449-3454 (1987)

35. Pace, C. N. Trends biochem. Sci. 15, 14-17 (1990).

36. Laskey, R. A.. Honda, B. M., Mills, A. D. \& Finch, J. T. Nature 275, 416-420 (1978)

37. Ellis, J. Nature 328, 378-379 (1987).

38. Rothman, J. E. Cell 59, 591-601 (1989)

39. Pfanner, $N$ et at $J$ biol Chem 265 , in the press (1990).

40. Marshall, J. S., DeRocher, A. E., Keegstra, K. \& Vierling, E. Proc. natn. Acad. Sci U.S.A. 87, 374-378 (1990)

41. Rose, M. D., Misra, L. M. \& Vogel, J. P. Cell 57, 1211-1221 (1989)

42. Normington, K., Kohno, K., Kozutsumi, Y., Gething, M.-J. \& Sambrook, d. Cell 57, 1223-1236 (1989)

43. Munro, S. \& Pelham, H. R. B. Cell 46, 291-300 (1986)

44. Waegemann, K., Paulsen, H. \& Soll, J. FEBS Lett. 261, 89-92 (1990)

45. Beckmann, R. P. Mizzen, L. A. \& Welch, W. J. Science 248, 850-854 (1990)

46. Bole, D. G., Hendershot, L. M. \& Kearney, J. F. I Cell Biol. 102, 1558-1566 (1986)

47. Copeland, C. S., Doms, R. W., Bolzau, E. M., Webster, R. G. \& Helenius, A. J. Cell Biol. 103 1179-1191 (1986) 
48. Gething, M.-J., McCammon, K. \& Sambrook, J. Cell 46, 939-950 (1986) 49. Pelham, H. R. B. Cell 46, 959-961 (1986).

50. Hendershot, L., Bole, D., Köhler, G. \& Kearney, J. F. J. Cell Biol. 104, 761-767 (1987).

51. Kassenbrock, C. K., Garcia, P. D. Walter, P. \& Kelly, R. B. Nature 333, 90-93 (1988)

52. Vogei, J. P. Misra, L. M. \& Rose, M. D. J. Cell Biol. 110, 1885-1895 (1990).

53. Nicchitta, C. V. \& Blobel, G. Cell 60, 259-269 (1990)

54. Flynn, G. C. Chappell, T. G. \& Rothman, J. E. Science 245, 385-390 (1989).

55. Phillips, G. J. \& Silhavy, T. J. Nature 344, 882-884 (1990).

56. Pfanner, N., Hartl, F.-U. \& Neupert, W. Eur. J. Biochem. 175, 205-212 (1988).

57. Keegstra, K. Cell 56, 247-253 (1989).
58. Nicolet, C. M. \& Craig, E. A. Molec. cell. Biol. 9, 3638-3646 (1989).

59. Kessler, S. W. Meth. Enzym. 73, 442-459 (1981).

ACKNOWLEDGEMENTS. We thank A. L. Horwich and M. Y. Cheng for the mif1 strain and for communicating unpublished results, and B. Guiard, R. L. Hallberg. B. Hennig and M. P. Yaffe for antisera directed against yeast cytochrome $b_{2}$, hsp 60 , cytochrome $c$ and $F_{1} \beta$, respectively. This work was directed against yeast cytochrome $b_{2}$, hsp60, cytochrome $c$ and $F_{1} \beta$, respectively. This work was
supported by the NHH (E.A.C.), the Deutsche Forschungsgemeinschaft and the Fonds der Chemischen supported by the NIR (E.A.C.), the Deutsche Forschungsgemeinschaft and the Fonds der Chemischen
Industrie. P.J.K. was supported by the Korean Ministry of Education and the Lucille P. Markey Charitable Trust.

\section{LETTERS TO NATURE}

\section{The potential distribution around growing fractal clusters}

\author{
Benoit B. Mandelbrot ${ }^{*}+\&$ Carl J. G. Evertsz \\ * Physics Department, IBM T. J. Watson Research Center, \\ Yorktown Heights, New York 10598, USA \\ $\dagger$ Mathematics Department and $\ddagger$ Applied Physics Department, \\ Yale University, New Haven, Connecticut 06520, USA
}

THE process of diffusion-limited aggregation (DLA) is a common means by which clusters grow from their constituent particles, as exemplified by the formation of soot and the aggregation of colloids in solution. DLA growth is a probabilistic process which results in the formation of fractal (self-similar) clusters. It is controlled by the harmonic measure (the gradient of the electrostatic potential) around the cluster's boundary. Here we show that interactive computer graphics can provide new insight into this potential distribution. We find that points of highest and lowest growth probability can lie unexpectedly close together, and that the lowest growth probabilities may lie very far from the initial seed. Our illustrations also reveal the prevalence of 'fjords' in which the pattern of equipotential lines involves a 'mainstream' with almost parallel walls. We suggest that an understanding of the low values of the harmonic measure will provide new understanding of the growth mechanism itself.

The DLA model of Witten and Sander ${ }^{1}$ captures the essential fractal aspects of a wide range of physical phenomena ${ }^{2-7}$, such as particle aggregation, dielectric breakdown, viscous fingering and electrochemical deposition. In DLA growth, an 'atom' executes Brownian motion until it hits, and becomes attached to, the curve that bounds a given 'target' or 'seed'. Another atom is then launched, and the whole process repeats. Initially, the growth rule and the pattern are very simple, but both become extremely complex in time. The distribution of hitting points of the atoms on the boundary of a fixed pattern becomes increasingly irregular and complex with repeated application of the rules. Simultaneously, the pattern becomes more complex (Fig. 1). Overwhelming evidence from computer simulations ${ }^{1-7}$ indi- cates that these patterns are near self-similar fractals ${ }^{8}$, meaning that their complication is about the same at all scales of observation that are sufficiently above the scale of the atom.

The distribution of the hitting points on the target's boundary is the harmonic measure, $\mu$ (ref. 9). This measure is obtained by solving the Laplace equation for the (electrostatic) potential, the boundary of the cluster being put at zero potential and a 'circle at infinity' being put at unit potential. The gradient of the potential at a point $P$ of the boundary defines the harmonic measure $\mu(P)$ at $P$. It is approximated by the potential at the lattice point nearest to $P$ (ref. 10), and is normalized to add up to one. The study of the laplacian potentials and their harmonic measures has been enriched by the example of DLA, because the process creates its own boundary conditions. The difficulties encountered in analytical approaches have made DLA a challenge in theoretical physics ${ }^{6,7,11-14}$ and in pure mathematics.

Figures 1, 2 and 3 show half of one of the many clusters we have grown on a cylindrical lattice, the initial target being the cylinder base. The many clusters drawn on a lattice in the original circle geometry ${ }^{1,10}$, in which the initial target is a cell in a square lattice, look similar (see cover figure). The cylindrical cluster shown in Figs 1-3 was grown to a height equal to its base $L=512$, and contains $\sim 30,000$ atoms. Once the cluster is obtained, the custom has been to solve the discrete Laplace equation ${ }^{10}$ on the original lattice on which DLA has been grown. But in the resulting illustrations, the potential cannot be seen with sufficient resolution in the many narrow 'fjords'. We therefore solved the Laplace equation on a lattice that was twice as fine. This equation was solved iteratively and the relative computation error was $\sim 0.01$.

Figure 1 and and the cover figure illustrate our first finding. As is usual in this field ${ }^{4}$, each atom is coloured to indicate the time it joined the cluster. In the background, rendered in black, the potential is above a certain threshold. Elsewhere, the 'whiteness' of the background increases with - $\log$ (potential). The low-potential zones look like ghostly 'cobwebs' extending to spatial scales larger than the atoms. Many occur in the bottom of fjords that may be short, but are narrow. Their location is very sample-dependent, and in many clusters they appear
FIG. 1 'Cobwebs' of very low potentials near a cylindrical cluster of diffusion-limited aggregation. The vertical cylinder base is to the left.

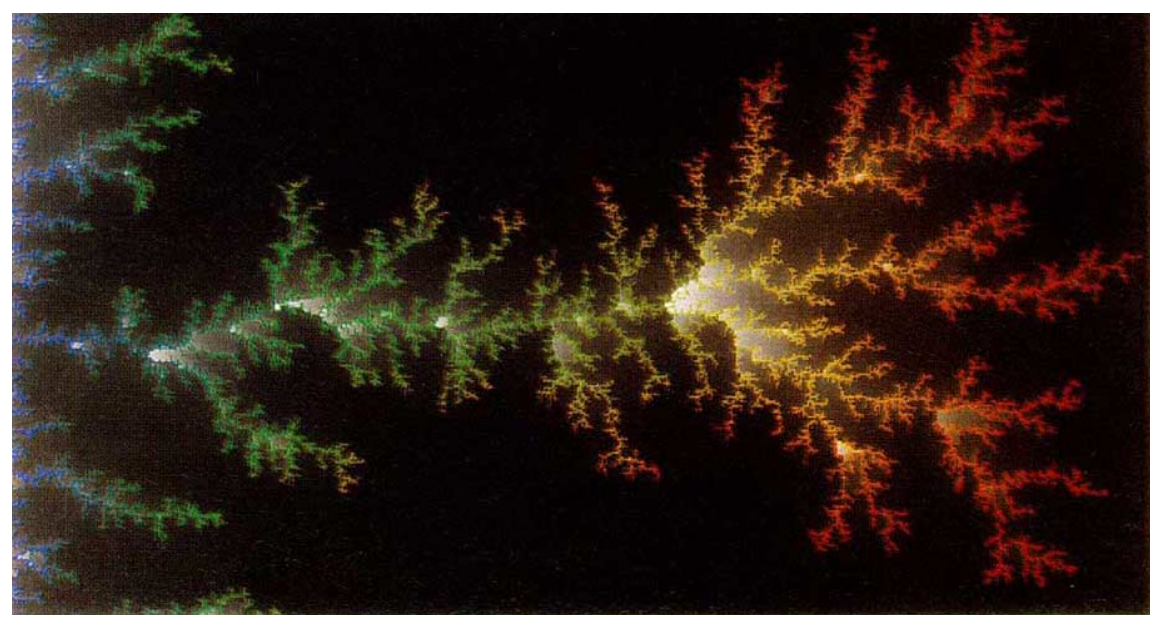

